Check for updates

Cite this: RSC Adv., 2017, 7, 47614

Received 19th August 2017

Accepted 29th September 2017

DOI: 10.1039/c7ra09170b

rsc.li/rsc-advances

\section{Structured growth from sheaf-like nuclei to highly asymmetric morphology in poly(nonamethylene terephthalate) $\uparrow$}

\begin{abstract}
C. H. Tu, E. M. Woo* and G. Lugito (iD)
Thorough microscopy analyses are done on dissecting 3D interiors of poly(nonamethylene terephthalate) (PNT) banded spherulites. Growth of a specific type (Type-I) of banded spherulites is initiated from highly elongated sheaf-like nuclei, with asymmetrically grown lamellae packed as a multi-shell structure, leading to resemblance of a dual-face spheroid. Top-surface bands are characterized with discrete/ unconnected dots of crystals aligned as zig-zag circles, which correspond to interior layered lamellae ends surfacing to top. Thus, the banded spherulites display not only inter-band discontinuity, but also discontinuity among the dotted crystals aligned on the top-surface ridges. 3D analysis exhibits that the banded spherulites possess a Janus-face morphology of compounded shell-and-kink lamellae assembly with dual discontinuities.
\end{abstract}

Organic or inorganic compounds, most notably calcium carbonate $\left(\mathrm{CaCO}_{3}\right)$, can react in a suitable regulating medium to form products of crystals displaying specific or diversified periodic assemblies on surfaces that are termed as surface-relief patterns that are intensively studied as model bio-mimetic systems. ${ }^{1-9}$ Additionally, small-molecule organic compounds such as phthalic acids have been reported to form periodic surface-relief patterns (concentric bands) upon solution evaporation induced crystallization. ${ }^{\mathbf{1 0 - 1 2}}$ Many organic polymers have also been known to display periodic ring patterns in meltcrystallized spherulites since the 1950's; however, albeit possessing similar or same periodic bands as those surface-relief patterns in small-molecule compounds, ${ }^{\mathbf{1 - 9}}$ the mechanisms and formation of the ring bands have been interesting and intriguing subjects in numerous studies for past many decades; yet, the subjects in polymer periodic patterns have remained to be argumentative for half a century, partially owing to an extra structural difference of existence of chain folding in the longchain backbones of polymers. Periodic ringed structures in spherulites of polymers, copolymers, or their blends have been discovered, studied and debated for a long time. ${ }^{13-22}$ Numerous re-visits or revisions of the model of continuous lamellar twisting for periodic banding have been attempted recently by investigators. ${ }^{23,24}$ Lamellar helix-twisting $360^{\circ}$-angle continuously like DNA molecules, had been one of the most often suggested mechanisms in explaining how crystal lamellae grow

Department of Chemical Engineering, National Cheng Kung University, Tainan, 701-01, Taiwan.E-mail: emwoo@mail.ncku.edu.tw

$\dagger$ Electronic supplementary information (ESI) available: Experimental detail, ${ }^{1} \mathrm{H}$ NMR, FTIR, GPC, and DSC characterization on the synthesized PNT. See DOI: 10.1039/c7ra09170b into periodic bands, as viewed in polarized microscopy. ${ }^{25-29} \mathrm{In}$ past decades, many investigators attributed the model of DNAlike continuous helix twisting of lamellae to surface stresses caused by chain-tilting in repeated folding; some other investigators even claimed that the twisting of polymer lamellae may be driven by polymer molecular chirality, which can be considered as a special form of chain tilting. ${ }^{30-35}$ The same phenomenon is also found in poly(trimethylene terephthalate) (PTT) that banding is attributed by some investigators to be due to chain tiling in repeated folding in the lamellae by Ivanov et al. in 2012. ${ }^{36}$ However, more recent re-investigation in 2017 on the bulk interiors of banded PTT spherulites $\left(T_{\mathrm{c}}=210{ }^{\circ} \mathrm{C}\right)$ has contested with proven evidence that interior assembly reveals that there are three banded PTT spherulite types, each originating from different nuclei geometries and corresponding to different assemblies of interior lamellae, but all possess similar multi-shell spheroid-layered structures, each with their layer thickness exactly equal to the optical inter-band spacing. ${ }^{37}$ This is to say, the interior lamellae are structured as polycrystalline and complex hierarchical forms and definitely not singlecrystalline plates in three types of banded PTT spherulites, and not just a single type of bands, essentially are not described by continuous helix-twisting, but by periodically interrupted interfaces between layered shells of spheroids. As the interior lamellae are not in accordance with continuous helix-twisting, chain tilting or folding-induced stresses is not at work for the optical banding in PTT spherulites.

Most polymers usually display a single type of ring-band pattern when crystallized at a $T_{\mathrm{c}}$. PNT upon crystallization is unique in that it can display simultaneously three different types of ring bands at a same $T_{\mathrm{c}}$. These three different ring- 
banded patterns (termed as: Type-0, -I, and -II) and association with crystallization kinetics in PNT have already been discussed in a couple of previous studies, ${ }^{38,39}$ where "Type- 0 band" means its lamellar assembly responsible for bands in surface is optically non-birefringent in POM; yet crystals of bands are visible in SEM. In earlier studies, PNT banded spherulites were mainly characterized for morphology of top surfaces in cast thin films with some interior lamellae being tentatively probed. ${ }^{38,39}$ Morphologies of Type-0, Type-I and Type-II spherulites in PNT thin films cast on glass slides and meltcrystallized at $60-85{ }^{\circ} \mathrm{C}$ have been expounded. Type-0 spherulites are superficially ringless in POM owing to ordered nanosize tiny ring structures; however, when viewed under greatermagnification SEM actually, the Type- 0 bands are patterned with thin and tiny dotted crystals (tens of nanometers) selfarranged as rings on top surfaces. Type-I and Type-II patterns in PNT, on the other hand, are apparently ringbanded but of different types as viewed under POM as well as SEM. The essential morphology difference of Type-I (singlebanded in POM with narrower inter-band spacing) versus Type-II (double-banded in POM with wider inter-band spacing) spherulites is obvious in SEM images. However, it is not enough to conceive the formation of ring bands if one only characterizes only the surface topography of thin films cast from solution on glass slides. Previous studies dealt with PNT in cast thin-film forms only, and 3-D patterns of ring bands of various types (Type-0, -I, and -II) in interiors of bulk forms were yet to be investigated. As the three types of bands differ significantly on the top-surface patterns, their corresponding interior assembly of lamellar crystals must differ in some subtle ways. Especially, three-dimensional views on interior assembly in correlation with top-surface relief patterned structures are necessary by specifically characterizing on intended types of banded PNT spherulites.

Although ring bands in thin-film polymers have been widely investigated and reported in the literature, 3-D ring pattern and growths, especially asymmetric ones and asymmetric-growth resulted interior crystal structures, have not been disclosed in any depth. Most studies on nuclei effects on crystal morphologies in the literature are limited to discussing the crystal growth confined in thin films without digging into interiors. An earlier study attempted to investigate the inner assembly of Type-I $v s$. Type-II ring bands in PNT spherulites..$^{40}$ Other than proposals that Type-I bands are more likely to be homogeneously nucleated and Type-II bands are more sensitive to external nucleation; however, detailed mechanisms responsible for the formation of Type-I $v s$. TypeII bands remained as a puzzle up to date. In this study, growth from nucleation stage till final crystallized Type-I bands were investigated in greater details using novel interior dissection approaches $v s$. observation of early-stage nuclei morphology and directional orientation. The mechanisms and structure evolution into Type-I band morphology with high asymmetry in interior banding structures of PNT spherulites were examined in this study. Alternatively, mapping of interior lamellae assembly in correlation with top-surface periodic banding of PNT Type-I bands was attempted in order to achieve a three- dimensional description of a peculiar type of ring bands spherulites that display high asymmetry in banding interior with zig-zag ordered top surface rings.

According to the earlier studies, ${ }^{38-40}$ PNT upon crystallization at $T_{\mathrm{c}}=85^{\circ} \mathrm{C}$ develops two types of ring-banded spherulites with totally different top surface-relief periodicity and patterns. It is quite unusual and complex that PNT can display simultaneously two different types of ring bands at a single $T_{\mathrm{c}}=85^{\circ} \mathrm{C}$ that differ in surface-relief patterns and likely also differ in interior lamellae packing; thus, mechanisms of co-existence of two types of ring bands may puzzle most investigators. Fig. 1 shows characteristic birefringence patterns in POM micrographs of Type-I $v s$. Type-II ring bands in PNT (at $T_{\mathrm{c}}=85^{\circ} \mathrm{C}$ by quenching from $T_{\max }=120^{\circ} \mathrm{C}$ ). Polarized optical micrographs (with or without tint plates) are placed side-by-side for direct evidence of apparent birefringence and morphological differences between these two types of ring bands. Fig. 1e shows that PNT crystallized at $T_{\mathrm{c}}=85{ }^{\circ} \mathrm{C}$ (after melt-heating at $T_{\max }=$ $110{ }^{\circ} \mathrm{C}$ ) displays a quantitative fraction ratio of Type-I/Type-II $=$ $3: 1$. Thus both types of ring bands can be easily identified. Type-I band is narrowly spaced (inter-ring $=7-8 \mu \mathrm{m}$ ) and zigzag ordered; while Type-II band is widely spaced (inter-ring $=$ $\sim 15-20 \mu \mathrm{m}$ ) and more orderly rings. In addition, it is quite common to have straight band-dislocation lines (as marked in white dashed-line brackets) in Type-II bands, but dislocation is



Fig. 1 POM graphs [with or without tint plates] for dual types of ringbanded spherulites of PNT: (a, b) Type-I spherulite (single ringbanded); (c, d) Type-II spherulite (double ring-banded) (scale bar = $20 \mu \mathrm{m}$ ); (e) lower magnification graph showing fractional distributions of Type-I vs. Type-II. 
never seen in Type-I bands. Reasons will be expounded in later analyses.

An earlier study using AFM focused only on top-surface morphology of both Type-I and Type-II banded PNT spherulites, and revealed the essential differences between these two types of bands; ${ }^{\mathbf{4 1}}$ however, how these top-surface banding patterns are correlated with respective interior lamellae assembly remained unknown. Fig. 2 shows comparison and confirmation of the dot-like zig-zag crystals on the top surfaces of Type-I PNT bands as viewed using both AFM and SEM, and these zig-zag dots are mostly in dimensions of micrometerscales, yet some substructures may be in nanometers. The AFM images of two different magnifications (Fig. 2a and b) show that dotted crystals are aligned into a rough circular bands on top surfaces of crystallized PNT film. The dotted crystals on bands have some fibrous tails dragging in radial direction, and these dotted crystals are not solidly connected in bands but are discretely separated from each other with micro- to nano-size gaps. AFM characterization results have proved that the zigzag aligned dotted domains on surface-relief patterns of TypeI bands are distinctly different from the much more elongated and better aligned rod-like crystals on surface-relief patterns of Type-II bands. ${ }^{42}$ Fig. $2 \mathrm{c}$ and $\mathrm{d}$ further show SEM micrographs for morphology differences on the spherulite center region of topsurface of Type-I bands. Agreement between the AFM and SEM results for the top-surface patterns for Type-I PNT bands is obvious. With the nuclei sites buried in depth of films, the top surface patterns are filled with fibrillar ends protruding from inner nuclei sheaf-crystals (Fig. 2c); with nuclei located near or on the top surface of films, the surfaces are more contrasted with dots of crystals aligned as zig-zag rings (Fig. 2d). Interior lamellae assembly corresponding to these various surface relief patterns, however, remains unknown until the banded

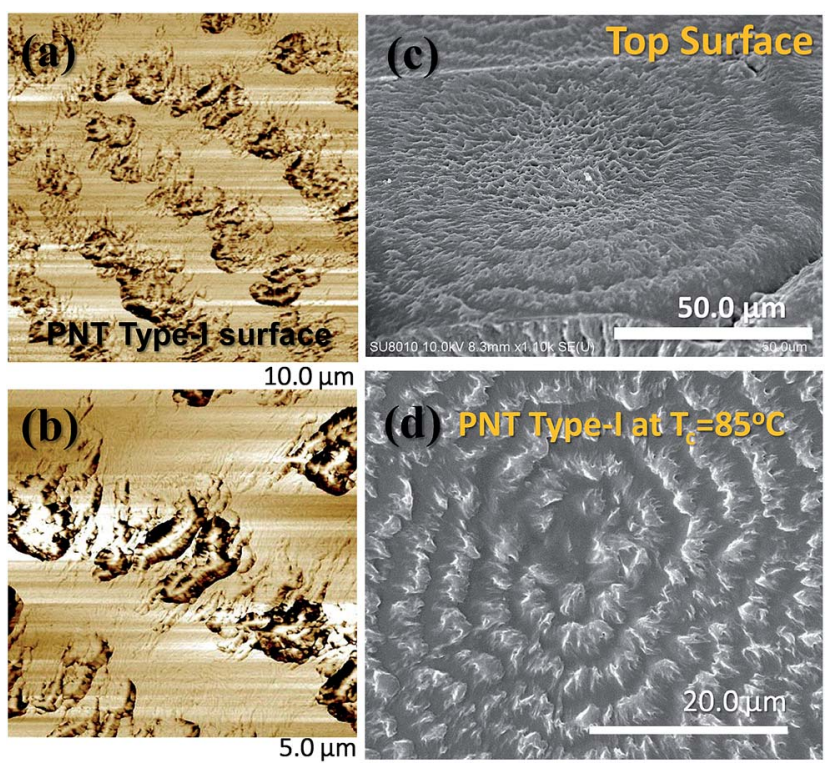

Fig. 2 (a, b) AFM images low lower and higher magnifications, respectively, and (c, d) SEM micrographs for different nuclei locations in PNT films crystallized at $T_{\mathrm{C}}=85^{\circ} \mathrm{C}$. spherulites can be opened for interior dissection analysis. It is critical to show correlation between the top-surface banding patterns with the interior lamellae and their assembly underneath these top-surface bands.

In further SEM characterization for interior dissection, the specimen containing Type-I PNT banded spherulites was placed on a metal stand with the specimen's fractured surface being oriented at a slant angle to the electron beams so that both top surface bands and fractured interior lamellae could be imaged simultaneously. Fig. 3 shows SEM micrographs of (a) topsurface banding of Type-I spherulites and interior lamellae that had been circumferentially fractured along the circular rings. For higher magnification $(5000 \times)$, (b) a region near the periphery of the banded PNT spherulite was zoomed-in to the square area on (a). Apparently, if one looks at the top surface of the spherulites, there are up-and-down alternating ring-bands; furthermore, if ones examine along the circumferential rings (Fig. 3b), there are alternating fan-like layer along the ring bands with distinct discontinuity. This is to say, not only there is an alternating pattern of circular concentric rings sharing a common center, but also there is alternating periodicity with discrete fan-like layers aligned along the circular rings. Each of the interior fan-like layers corresponds to a discrete dot on the top surface. The dots on the top surface are actually protrusions of these inner fan-like lamellae and these dots are loosely and zig-zag aligned on the top surface to appear as "bands". Nevertheless, underneath these dot-like bands on top surface, lamellae are aligned as highly palm-branched fans, and there is no $360^{\circ}$-angle helix-twisting of lamellae. These fan-like lamellae collectively wave and branch out periodically from an inner center (bottom center of Fig. 3a).

Even though the subject polymer has a relatively low melting point $\left(92^{\circ} \mathrm{C}\right)$ that may result in some electron charging issues in SEM; it was still possible to manage to capture considerably high-resolution images to show the details of lamellar assembly in the specimens of PNT banded spherulites. Fig. 4 shows a schematic illustration for the top-surface ridges of Type-I spherulite in correlation with the corresponding interior lamellae assembly. Apparently, not only the top surface banding displays periodic bands, the interior fan-like lamellae are accompanied with periodic tilting/bending and branching, and the fan-like lamellae are all aligned underneath and along the periphery of circular bands. The interior periodicity of bending/



Fig. 3 SEM micrographs of Type-I spherulites being circumferentially (along the rings) fractured: (a) region near periphery of banded spherulite (2000 X), (b) zoom-in to the square area on (a) (5000x). 

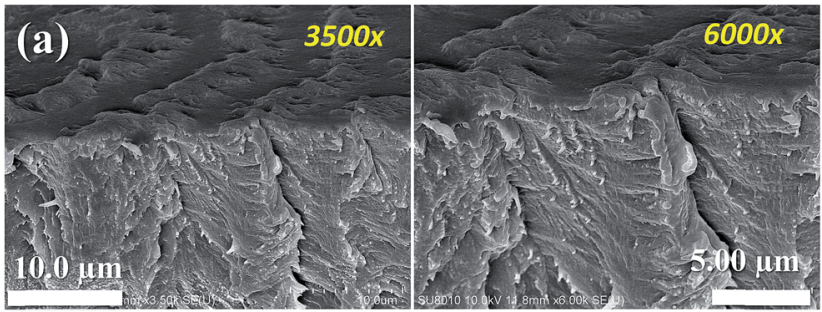

(c)

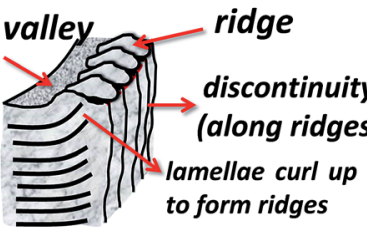

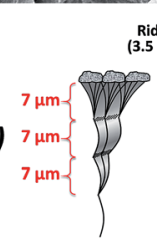

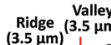

Top
center

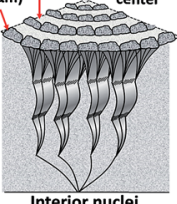

Fig. 4 SEM micrograph and schematic illustration for top-surface band ridges of Type-I spherulite vs. corresponding interior lamellae accompanied with periodically-tilt arrangements (along the periphery of circular bands).

branching is $c a .7 \mu \mathrm{m}$, which is roughly same as the inter-ring spacing on top surface $(7 \mu \mathrm{m})$, which both are exactly equal to the optical inter-band spacing $(7 \mu \mathrm{m})$. This interior-top surface analysis and correlation fully proves that optical banding of Type-I spherulites in PNT film is not attributed to $360^{\circ}$-angle helix-twisting of lamellae along the top surface of films, but due to periodic assembly of the highly branched inner lamellae undergoing periodic fanning out from a common nuclei center in bulk interior (i.e., homogeneous nucleation).
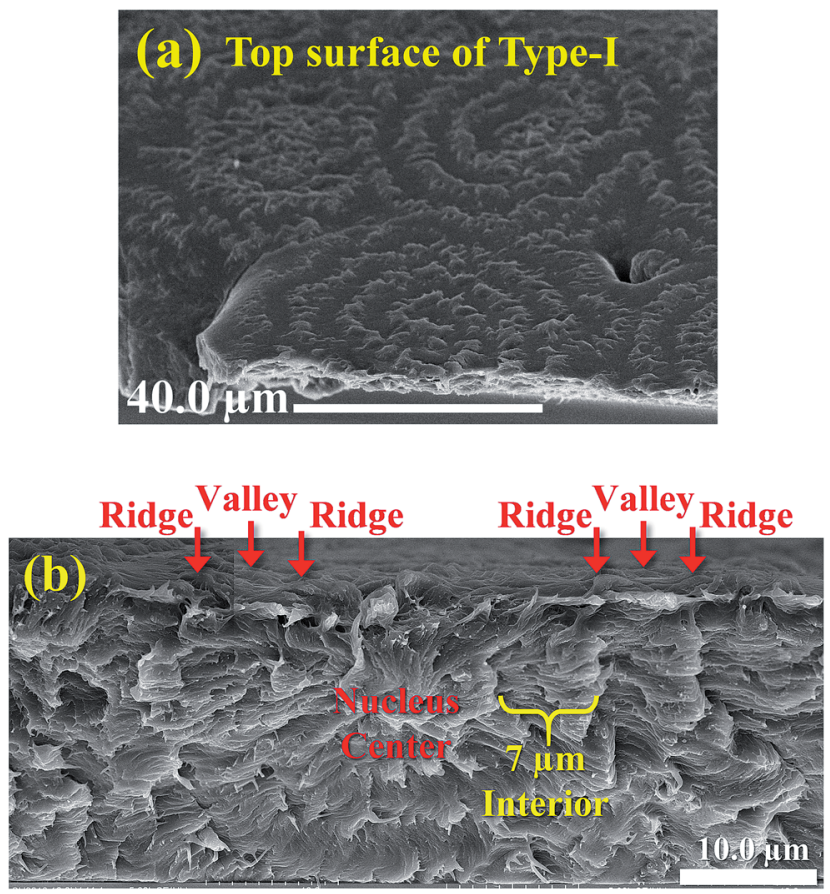

Fig. 5 SEM for interiors and schemes showing correlation between top surface and inner lamellae assembly of Type-I ring-banded PNT spherulite with nuclei located near interface (polymer/air).
It has been shown earlier ${ }^{39}$ that the fractured interiors of the banded PNT spherulites grown inside can show different patterns with the outside surface for both Type-I and Type-II spherulites, which is attributed to distortion of crystals upon reaching polymer-air interface. Nevertheless, there is a consistent correlation between the top-surface banding and interior lamellae assembly in terms of crystal lamellae emerging from interiors to outer surfaces. Schematic illustrations in Fig. 5 further show the interior dissection based on SEM analyses into the lamellae hidden underneath the Type-I PNT bands display alternating ridge/valley variation on top surface (band spacing $=7 \mu \mathrm{m}$ ), while the interior lamellae are self- assembled into a layer-like structure with a periodicity layer thickness $=7.0 \mu \mathrm{m}$. The periodicity in the inner lamellae is caused by characteristic curling, bending and branching at a fixed interval. Note also Fig. 5a shows fan-like lamellae are aligned along the circular bands with distinct discontinuity between the lamellae plates, where each lamellar plate measures a thickness $c a .1 \mu \mathrm{m}$. As discussed, the interior lamellae (underneath the top surface of spherulites) may inevitably differ from the top-surface morphologies of Type-I PNT bands. It further demonstrates that one runs great risk in probing the mechanisms of periodic banding in polymer spherulites if the investigator only constrains analyses on the top-surface morphology of thin crystallized films. In addition, nuclei crystals generated from homogeneous nucleation usually take a shape of elongated sheaf bundles, which lead to highly asymmetric growth into dual-face lamellae structures. Fig. 6a shows the nuclei of are located in inner bulk of Type-I banded spherulites $\left(T_{\mathrm{c}}=85^{\circ} \mathrm{C}\right)$ and take a sheaf-like geometry (slanted $45^{\circ}$-angle with the top surface). The lamellae grow along the sheaf direction are structured to a morphology as depicted, while in the perpendicular direction to the nuclei-sheaf, the crystals are packed into a kinked layered structure (scheme in Fig. 6b). The difference in morphology is likely caused by lamellar orientations; that is, along the sheaf, the lamellae are mostly flat-on (with the basal face pointing upward) but the lamellae in the perpendicular growth are edge-on oriented (with crystal prism face pointing out of the paper).
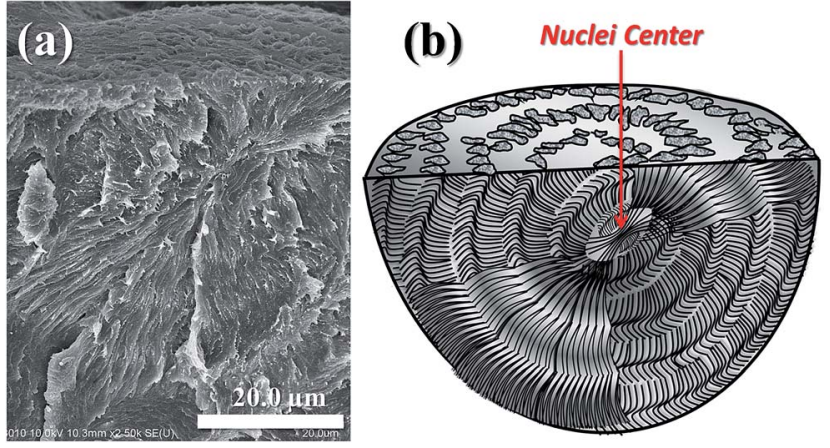

Fig. 6 Asymmetric growth from sheaf-nuclei in Type-I PNT ring bands: (a) SEM graph showing nuclei sheaf in bulk center of spherulite, (b) schematic for asymmetry in interior lamellae assembly around the nuclei in Type-I banded spherulite $(2500 x)$. 


\section{Conclusions}

Type-I asymmetric ring-banded PNT spherulites are nucleated from highly oriented sheaf-nuclei that lead to disproportional distribution of lamellae assembly into two half-regions of different periodic packing. The 3-D structure in a PNT Type-I banded spherulite resembles a dual-face spheroid. The multilayered structures, with periodic kinked curling of pitch = $7 \mu \mathrm{m}$, as revealed in SEM characterization on PNT Type-I bands stand for alternating growth textures from innermost to outside, except that that the exterior banding patterns are influenced by bulk-air interfacial stresses. The 3-D analysis thus exhibits an authentic cross-sectional morphology appearing as asymmetrically compounded half-and-half lamellae assembly.

\section{Conflicts of interest}

There are no conflicts to declare.

\section{Acknowledgements}

This work was financially supported by a basic research grant (MOST 105-2221-E-006-246-MY3) in three consecutive years funded by Ministry of Science and Technology (MOST) of Taiwan.

\section{Notes and references}

1 S. Mann and G. A. Ozin, Nature, 1996, 382, 313-318.

2 D. Walsh and S. Mann, Nature, 1995, 377, 320-323.

3 A. Sugawara, T. Ishii and T. Kato, Angew. Chem., 2003, 115, 5457-5461.

4 T. Kato, Adv. Mater., 2000, 12, 1543-1546.

5 H. Cölfen and M. Antonietti, Langmuir, 1998, 14, 582-589.

6 G. Xu, N. Yao, I. A. Aksay and J. T. Groves, J. Am. Chem. Soc., 1998, 120, 11977-11985.

7 J. Aizenberg, A. J. Black and G. M. Whitesides, Nature, 1999, 398, 495-498.

8 J. Aizenberg, A. J. Black and G. M. Whitesides, J. Am. Chem. Soc., 1999, 121, 4500-4509.

9 L. Qi, J. Li and J. Ma, Adv. Mater., 2002, 14, 300-303.

10 E. M. Woo, G. Lugito and C. E. Yang, CrystEngComm, 2016, 18, 977-985.

11 A. G. Shtukenberg, Y. O. Punin, E. Gunn and B. Kahr, Chem. Rev., 2012, 112, 1805-1838.

12 E. Gunn, R. Sours, J. B. Benedict, W. Kaminsky and B. Kahr, J. Am. Chem. Soc., 2006, 128, 14234-14235.

13 A. J. Keller, J. Polym. Sci., 1959, 39, 151-173.

14 H. D. Keith and F. J. Padden Jr, J. Polym. Sci., 1959, 39, 101122.

15 H. D. Keith and F. J. Padden Jr, J. Polym. Sci., 1959, 39, 123138.
16 Z. Wang, X. Wang, D. Yu and B. Jiang, Polymer, 1997, 38, 5897-5901.

17 Y. Okabe, T. Kyu, H. Saito and T. Inoue, Macromolecules, 1998, 31, 5823-5829.

18 L. G. M. Beekmans, M. A. Hempenius and G. J. Vancso, Eur. Polym. J., 2004, 40, 893-903.

19 Z. L. Cheung, L. T. Weng, C. M. Chan, W. M. Hou and L. Li, Langmuir, 2005, 21, 7968-7970.

20 J. Chen and D. Yang, Macromolecules, 2005, 38, 3371-3379.

21 H. Kajioka, A. Hoshino, H. Miyaji, Y. Miyamoto, A. Toda and M. Hikosaka, Polymer, 2005, 46, 8717-8722.

22 H. D. Keith and F. J. Padden Jr, Macromolecules, 1996, 29, 7776-7786.

23 J. M. Schultz, Macromolecules, 2013, 46, 4227-4229.

24 M. Rosenthal, G. Portale, M. Burghammer, G. Bar, E. T. Samulski and D. A. Ivanov, Macromolecules, 2012, 45, 7454-7460.

25 D. C. Bassett, R. H. Olley and I. A. M. Al Raheil, Polymer, 1988, 29, 1539-1543.

26 D. C. Bassett, Philos. Trans. R. Soc., A, 1994, 348, $29-43$.

27 Y. Jiang, J. J. Zhou, L. Li, J. Xu, B. H. Guo, Z. M. Zhang, Q. Wu, G. Q. Chen, L. T. Weng, Z. L. Cheung and C. M. Chan, Langmuir, 2003, 19, 7417-7422.

28 J. Xu, B. H. Guo, Z. M. Zhang, J. J. Zhou, Y. Jiang, S. Yan, L. Li, Q. Wu, G. Q. Chen and J. M. Schultz, Macromolecules, 2004, 37, 4118-4123.

29 S. Sasaki, Y. Sasaki, A. Takahara and T. Kajiyama, Polymer, 2002, 43, 3441-3446.

30 H. D. Keith, Polymer, 2001, 42, 9987-9993.

31 H. D. Keith and F. J. Padden Jr, Polymer, 1984, 25, 28-42.

32 D. Maillar and R. E. Prud'homme, Macromolecules, 2006, 39, 4272-4275.

33 D. Maillard and R. E. Prud'homme, Macromolecules, 2008, 41, 1705-1712.

34 K. L. Singfield, J. K. Hobbs and A. Keller, J. Cryst. Growth, 1998, 183, 683-689.

35 I. Saracovan, H. D. Keith, R. S. J. Manley and G. R. Brown, Macromolecules, 1999, 32, 8918-8922.

36 M. Rosenthal, M. Burghammer, G. Bar, E. T. Samulski and D. A. Ivanov, Macromolecules, 2014, 47, 8295-8304.

37 G. Lugito and E. M. Woo, Macromolecules, 2017, 50, 58985904.

38 Y. F. Chen, E. M. Woo and S. H. Li, Langmuir, 2008, 24, 11880-11888.

39 E. M. Woo and Y. F. Chen, Polymer, 2009, 50, 4706-4717.

40 Y. F. Chen and E. M. Woo, Macromol. Rapid Commun., 2009, 30, 1911-1916.

41 G. Lugito, E. M. Woo and S. M. Chang, J. Polym. Sci., Part B: Polym. Phys., 2017, 55, 601.

42 E. M. Woo and S. Nurkhamidah, J. Phys. Chem. B, 2012, 116, 5071. 